\title{
CHARACTERIZATION OF ABSTRACT COMPOSITION OPERATORS
}

\author{
WILLIAM C. RIDGE
}

ABSTRACT. A composition operator on $L^{p}(X, \mu)$ is (roughly) an operator $T$ induced by a point transformation $\phi$ on $X$ by $T f=f \cdot \phi$.

Characterizations are given of abstract Hilbert-space operators which can be represented (via unitary equivalence) as composition operators. Representation on $L^{2}(J, m)(J$ an interval of the real line, $m$ a Borel measure) and on $L^{2}(0,1)$ (Lebe sgue measure) are considered.

Also, any bounded measure-algebra transformation which preserves disjoint unions is a sigma-homomorphism.

Notation. Throughout this paper, $H$ denotes a Hilbert space of countably infinite dimension, and $T$ denotes a bounded linear operator on $H$.

Definition. An operator $T$ on $L^{p}(X, \mu)$ induced by a transformation $\phi$ : $X_{1} \rightarrow X, X_{1} \subset X$, by the formula $T f=(f \cdot \phi) \chi_{X_{1}}$ is called a composition operator.

Definition (Choksi [2, p. 89]). A subset $C$ of $H$ is a $C$-family if it satisfies the following conditions:

(1) $C$ is closed (topologically) in $H$.

(2) $C$ is total in $H$.

(3) $C$ has a unique element $i$ of maximal norm $\|i\|=1$.

(4) If $f \in C$, then $i-f \in C$ and $(f, i-f)=0$.

(5) If $f, g, h \in C$ and $(h, i-g)=(g, f)=0$, then $(h, f)=0$.

(6) If $f, g \in C$, then $f+g \in C$ if and only if $(f, g)=0$.

(7) If $f \in C$ and $f \neq i$, then $C$ has an element $g \neq 0$ such that $g \neq i-$ $f$ and $(f, g)=0$.

(8) If $f, g \in C$, then $C$ has a unique element $h$ such that $(h, i-f)=$ $(h, i-g)=0$ and $\|h\|^{2}=(f, g)$. $(f, g)$ is real and nonnegative.

Theorem (Choksi [2, p. 94]), A subset $C$ of $H$ corresponds to the char-

Received by the editors January 20, 1973 and, in revised form, May 28, 1973. AMS (MOS) subject classifications (1970). Primary 28A65, 47 A 10, 47 A35.

Key words and phrases. Linear operators, Hilbert space, measure algebra, measurable transformations, sigma-homomorphisms. 
acteristic functions under some isometry of $H$ with $L^{2}(0,1)$, Lebesgue measure, if and only if it is a C-family.

Definition (Sz.-Nagy). A subset $C$ of $H$ is an $N$-family if it satisfies the following conditions. We say $f<g$ if $(f, g)=\|f\|^{2}$.

(1) $C$ is total in $H$.

(2) If $f, g \in C$, then $f-g \in C$ if and only if $g<f$.

(3) For all $f, g$ in $C$, there are elements $h, k$ of $C$ such that $f+g=$ $h+k, h<f, h<g$.

(4) If $\left(f_{k}\right)$ is an increasing sequence from $C$ with a limit $f$ in $H$, then $f$ is in $C$.

Theorem (Sz.-Nagy). A subset $C$ of $H$ corresponds to the characteristic functions under some isometry of $H$ with $L^{2}(J, m), J$ a (finite or infinite) interval on the real line, $m$ a Borel measure, if and only if $C$ is an N-family

Definition. Let $\Phi$ be a transformation from a measure algebra $(A, \mu)$ to another, $(B, \nu)$. The norm of $\Phi$ is

$$
\|\Phi\|=\sup \{\nu[\Phi(\alpha)] / \mu(\alpha): 0<\mu(\alpha)<\infty, \alpha \in A\} .
$$

$\Phi$ is bounded if $\|\Phi\|<\infty$. $\Phi$ is additive if $\alpha \wedge \beta=0$ implies $\Phi(\alpha \vee \beta)=$ $\Phi(\alpha) \vee \Phi(\beta)$ and $\Phi(\alpha) \wedge \Phi(\beta)=0$.

Lemma. Every bounded additive transformation from a totally finite measure algebra into an arbitrary measure algebra is a sigma-homomorphism.

Proof. Let $\Phi:(A, \mu) \rightarrow(B, \nu)$ be bounded and additive, $(A, \mu)$ totally finite. For $\alpha, \beta$ in $A$,

$$
\Phi(\alpha)=\Phi(\alpha \wedge \beta) \vee \Phi(\alpha-\beta) \text { and } \Phi(\beta)=\Phi(\alpha \wedge \beta) \vee \Phi(\beta-\alpha),
$$

both disjoint unions, by additivity. Taking intersections on both sides, we see that $\Phi$ preserves (by induction) finite intersections. This, together with additivity, implies that $\Phi$ preserves differences, for

$$
\Phi(\alpha-\beta)=\Phi(\alpha)-\Phi(\alpha \wedge \beta)=\Phi(\alpha)-[\Phi(\alpha) \wedge \Phi(\beta)]=\Phi(\alpha)-\Phi(\beta) .
$$

Suppose $\alpha=$ (countable) $\bigvee a_{\kappa}$ with $\alpha, \alpha_{\kappa}$ in $A$. Since additivity implies monotonicity,

$$
\vee \Phi\left(\alpha_{\kappa}\right) \leq \Phi(\alpha) \text {. }
$$

If they are not equal, then $\nu\left[\Phi(\alpha)-\bigvee \Phi\left(\alpha_{\kappa}\right)\right]=d>0$.

For some integer $s, \mu\left(\alpha-\bigvee_{\kappa=1}^{s} a_{\kappa}\right)<d /\|\Phi\|$. 
Then

$$
\|\Phi\|>\frac{\nu \cdot \Phi\left(\alpha-V_{\kappa=1}^{s} a_{\kappa}\right)}{\mu\left(\alpha-V_{\kappa=1}^{s} a_{\kappa}\right)}>\frac{\nu \cdot \Phi\left(\alpha-V_{\alpha_{K}}\right)}{\mu\left(\alpha-\bigvee_{K=1}^{s} \alpha_{\kappa}\right)}>\|\Phi\|,
$$

a contradiction. So $\Phi$ preserves countable unions, and is hence a sigma-homomorphism.

Theorem 1. An operator $T$ on $H$ is unitarily equivalent to a composition operator on $L^{2}(0,1)$, Lebesgue measure, if and only if there is a $C$-family $C$ in $H$ such that $T C \subset C$.

Proof. The characteristic functions on $(0,1)$ do form a $C$-family, and every composition operator takes characteristic functions into characteristic functions.

Conversely, suppose $T C \subset C$ where $C$ is a $C$-family. By Choksi's theorem we may (by unitary equivalence) identify $H$ with $L^{2}(0,1)$, Lebesgue measure, and $C$ with the characteristic functions, with $T C \subset C$. If $(A, \mu)$ is the measure algebra (Lebesgue) of $(0,1)$ and $\alpha \in A$, then $T \chi_{a}$ is the characteristic function of an element $\Phi(\alpha)$ of $A$. This defines a transformation $\Phi$ on $A$.

If $\alpha \wedge \beta=0$, then

$$
\chi_{\Phi(\alpha \vee \beta)}=T \chi_{a \vee \beta}=T \chi_{a}+T \chi_{\beta}=\chi_{\Phi(a)}+\chi_{\Phi(\beta)}
$$

so

$$
\Phi(\alpha \vee \beta)=\Phi(\alpha) \vee \Phi(\beta)
$$

disjoint union, and so $\Phi$ is additive. Also

$$
\mu[\Phi(\alpha)]=\left\|\chi_{\Phi(\alpha)}\right\|^{2}=\left\|T \chi_{\alpha}\right\|^{2} \leq\|T\|^{2} \mu(\alpha)
$$

so

$$
\|\Phi\| \leq\|T\|^{2}
$$

and $\Phi$ is bounded. By the above Lemma, $\Phi$ is therefore a sigma-homomorphism, and by [6] there is a measurable point tran sformation $\phi: X_{1} \rightarrow(0,1)$ such that $\phi^{-1} E \in \Phi(\alpha)$ for all Borel sets $E$, where $\alpha$ is the equivalence class of $E$ in $A$, and $X_{1}$ is a measurable subset of $(0,1)$.

Let $T_{\phi}$ be the composition operator on $L^{2}(0,1)$ induced by $\phi, E$ any Borel set, and $\alpha$ its equivalence class in $A$. Then

$$
T \chi_{a}=\chi_{\Phi(a)}=\left[\chi_{\phi^{-1} E}\right]=\left[\chi_{E} \circ \phi\right]=T_{\phi} \chi_{a}
$$


where [ 0$]$ denotes equivalence class in $L^{2}$. Since $T_{\phi}$ is bounded by its bound on characteristic functions (a routine exercise, or see [4] or [5]), $T$ and $T_{\phi}$ are bounded linear operators which agree on characteristic functions, and hence $T=T_{\phi}$.

Theorem 2. An operator $T$ on $H$ is unitarily equivalent to a composition operator if and only if there is an N-family $C$ in $H$ such that TC C C.

Proof. Characteristic functions on a measure space do form an $N$-family, which again is always invariant under a composition operator.

If $T$ leaves an $N$-family invariant, then by Sz-Nagy's theorem $T$ is unitarily equival ent to an operator $S$ on $L^{2}(J, m)$ ( $J$ an interval, $m$ a Borel measure) which takes characteristic functions into characteristic functions. As in Theorem 1, $s \chi_{\alpha}=\chi_{\Phi(a)}$ where $\Phi$ is a sigma-homomorphism on the collection of sets having finite measure. (Restrict $\Phi$ to any finite subinterval and apply the above Lemma.) By the first corollary to Sikorski's theorem [6], there is a measurable transformation $\phi: J_{0} \rightarrow J$ with $\phi^{-1} E \in \Phi(\alpha)$ for all Borel sets $E, \alpha$ being the equival ence class of $E$ in the measure algebra of $J$, and $J_{0}$ being a measurable subset of $J$. Then $\phi$ induces $S$ as in the proof of Theorem 1.

Corollary. Any composition operator on a separable infinite-dimensional Hilbert space can be represented on an interval of the real line with a Borel measure.

\section{REFERENCES}

1. J. R. Choksi, Non-erodic transformations with discrete spectrum, Illinois J. Math. 9 (1965), 307-320. MR 30 \#4903.

2. - Unitary operators induced by measure-preserving transformations, J. Math. Mech. 16 (1966), 83-100. MR 34 \# 1844.

3. - Unitary operators induced by measurable transformations, J. Math. Mech. 17 (1967/68), 785-801. MR 36 \#2003.

4. W. C. Ridge, Composition operators, Thesis, Indiana University, Bloomington, Ind., 1969, pp. 1-11, 17-22.

5. - Spectrum of a composition operator, Proc. Amer. Math. Soc. 37 (1973), 121-127.

6. R. Sikorski, Boolean algebras, 2nd ed., Ergebnisse der Mathematik und ihrer Grenzgebiete, N. F., Band 25, Academic Press, New York; Springer-Verlag, Berlin, 1964, P. 139. MR 31 \#2178.

7. B. Sz.-Nagy, Über die Gesamtheit der charakteristischen Funktionen im Hilbertschen Funktionenraum, Acta Sci. Math. (Szeged) 9 (1937), 166-176.

DEPARTMENT OF MATHEMATICAL SCIENCES, INDIANA UNIVERSITY-PURDUE UNIVERSITY, INDIANAPOLIS, INDIANA 46205 